\title{
EDITORIAL Importance of Business Continuity in Health
} Care

\author{
Arnold B. Bierenbaum, MS, Beth Neiley, RN, MS, and Craig R. Savageau, MS
}

$\mathrm{I}$ is 11:30 PM on a hot and humid night. Your hospital was just informed that the municipal power supply has been disrupted, with no estimated restoration time. No problem, right? That is what emergency power is for.

Another call comes in informing you that patients are complaining of being uncomfortably warm. Although the power in the hospital never went out, is there a relationship between the increased room temperature and the municipal outage? Is there a bigger problem? What is the hospital's continuity plan? If you know the answer to the above questions, then you probably appreciate the importance of continuity planning. If not, consider reading on.

The hurricane seasons of 2004 and 2005 taught health care leaders that developing, managing, and maintaining continuity of operations plans is crucial. Of particular importance is the ability for a hospital to sustain mission critical systems in the presence of a major disruption (ie, electrical power failure, water loss). This consistent ability to sustain critical systems is one of the main decision points in determining whether a hospital should shelter in place or be evacuated.

The Florida Hospital Association conducted a statistical analysis of the impact hurricanes had on Florida hospitals during the 2004 hurricane season. Of 93 hospitals surveyed, 41 reported losing electrical power. An additional 9 reported loss of water supply and 11 reported losing some other type of critical support function. ${ }^{1}$

As health care facility leadership prioritize the need for enhancing critical infrastructure, emphasis should be placed on the overall role of the facility. Health care facilities not only treat and care for the injured and ill but they also provide a sense of stability for the community during and after hazard impact. ${ }^{2}$

Clinicians contributing to emergency planning often overlook the crucial nature of essential services and the impact of service disruptions. This editorial focuses on the mission critical systems that help to sustain patient care, including electrical power; water supply; heating, ventilation, and air conditioning (HVAC); and information technology (IT) resiliency. In addition, the authors will discuss 9 steps that hospitals should consider as part of their business continuity planning activities.
One of the most critical systems in a health care facility is the electrical power supply. Most nonengineers do not consider electrical power in their day-to-day activities, but its impact is felt after it fails. In addition, many hospital managers and leaders do not consider that in most facilities, emergency electrical generators, used as backup sources for electricity, do not provide power to all areas of the hospital. Examples of critical areas not frequently considered for backup power include elevators, necessary for ensuring vertical evacuation of critical care patients, ${ }^{3}$ and "ancillary areas, such as the morgue." ${ }^{1}$ In addition, electrical power redundancy ensures that equipment is operational to support critical care patients maintained on life support. Thus, a hospital could be completely compliant with The Joint Commission (TJC) and other standards that require a backup generator, and still face significant operational risks should power be disrupted.

Another important critical system is water supply. A tertiary care hospital could consume more than 100,000 gal/day. Water is needed for drinking, bathing, ice, cleaning, waste disposal, heating, and air conditioning. The availability of drinking water within the first few days of hazard impact could affect the decision to shelter in place versus evacuate.

Mitigation plans may include stockpiling bottled water or installing a water tower and/or wells (depending upon location). Regardless of the actions in the mitigation plan, possessing some form of alternate water supply may be the difference between a hospital remaining open or needing to be evacuated. Hospitals should also consider having a priority resupply contract for water in the event that the stockpile on hand is reduced or deleted from use. Hospital leaders must also understand that indirect hazard impact could have an affect on their facility. During Hurricane Katrina, some hospitals that were not affected by direct flooding were without water for up to 10 days due to the damage to water infrastructure of New Orleans. ${ }^{3}$ Katrina showed that hospitals depend heavily on citywide infrastructure-electrical power, communications, water, security, and transportation-which can be disrupted by an areawide disaster. ${ }^{4}$

Hospital planners also must ensure the resiliency of HVAC systems. Air flow control to establish negative pressure for isolation from external contamination resulting from industrial accidents or terrorist events should be considered when 
evaluating HVAC capability. During the 2004 and 2005 hurricane seasons, hospitals found themselves facing a unique threat while sheltering in place: the elevation of temperatures due to lack of backup generators that controlled the HVAC systems. In addition, without the use of HVAC, a hospital is susceptible to mold growth. ${ }^{3}$

Because hospitals increasingly rely upon IT for business as well as patient care operations, careful continuity planning is required, with multiple contingencies including emergency power, communications, mirror computing sites, and off-site data storage. Maintaining communications during disasters can prove to be especially difficult. According to the Florida Hospital Association's 2005 report, the loss of communication capability during the 2004 hurricane season increased its awareness of the need to bridge the communication gap between hospitals and their communities. ${ }^{1}$ In 1 incident during the aftermath of Hurricane Katrina, a hospital employee's husband stood on the roof of the hospital and transmitted a "mayday" alert through his ham radio to anyone who was listening. ${ }^{3}$

Although disruption to these 4 mission critical systems can bring activities within a hospital to a halt, there are many other systems that must be addressed, including medical gases, vacuum, oxygen, fire alarms, sprinklers, public address, and waste disposal.

According to the International Strategy for Disaster Reduction and the World Health Organization's 2008-2009 World Disaster Reduction Campaign, "The price we pay for the failure of hospitals or health care facilities due to disasters is too high. In comparison, the cost of making hospitals safe from disasters is tiny." 2

Strengthening the infrastructure through continuity planning provides the basis for comprehensive emergency management. The cost-benefit ratio for building and enhancing health care systems' infrastructures competes, at times, with many other facility projects. Although the cost for implementing protective measures into the construction of new health facilities increases costs about $4 \%$ overall, "retrofitting nonstructural elements" increases costs by only $1 \%$.

In 2007, the Veterans Health Administration partnered with the consulting firm Booz Allen Hamilton to assess the status of emergency management programs across the system. To conduct these assessments, evaluation teams visited Department of Veterans Affairs (VA) medical centers across the nation and conducted facilitated group interviews, individual interviews, tabletop exercises, and capability demonstrations in an attempt to understand the emergency preparednessrelated capabilities of VA medical centers. The assessment teams were particularly impressed with the mitigation and contingency planning of hospitals located in Florida and the Caribbean.

VA hired an architectural/engineering firm in 2004 to determine how to improve the resiliency of mission critical sys- tems in all facilities at risk for hurricanes, including all of the VA medical centers in Florida and Puerto Rico. The Veterans Integrated Service Network identified $\$ 67$ million to strengthen mission critical systems by mitigating a number of the highest risk hazards to these systems. More than \$34 million of these mitigation activities have been funded and are in various stages of design and construction. Once completed, these will give the medical facilities the abilities to maintain electric, water, HVAC, IT, and many other mission critical systems for approximately 7 days.

So, what should the hospital consider? In what order? The following is a 9-step process that begins with a basic analysis of the present capabilities and an identification of known gaps in coverage or infrastructure deficiencies. Then, after the limitations and potential weaknesses in the system are identified, plans can be put in place to improve the issues, train staff, conduct exercises, and evaluate the results. This will lead to a high-quality approach to developing contingency and continuity plans.

1. Know the capabilities and limitations of the institution's mission critical systems. The first and most critical step is for all department heads and senior hospital management (including clinical leadership) to be aware of these capabilities and limitations. All too often, this understanding is limited to the hospital engineer or facility manager.

2. Ensure that a risk assessment is conducted for mitigation projects. These projects should be based on the hazard vulnerability analysis, as required by TJC, and should ensure that activities necessary to mitigate effects from specified hazards are prioritized. It is important to consider the impact that the surrounding community may have upon the hospital's critical infrastructure in a disaster such as a release of toxic materials from a neighboring industrial facility. Once the hospital administration and clinical department heads are aware of the capabilities of their mission critical systems, such as emergency power capabilities, contingency plans can be made by each department to minimize the risks and sustain operations. For example, mitigation plans can be developed to increase the capabilities of mission critical systems by adding additional capacity or securing additional resources via contract should they be needed.

3. Develop mitigation plans and projects to enhance resiliency. Hospital leaders and their emergency planners should develop well-thought-out mitigation plans that will ensure continued operations in times of outage. Examples include capability to provide 100\% auxiliary power, operate computerized energy control systems, and access campus water towers.

4. Develop contingency plans for the hospital and each department should the mission critical systems fail. Medical and hospital operations leaders should carefully consider developing multiple layers of contingency plans as backup in the event of loss of mission critical systems or failure of mitigation plans. Examples include water con- 
servation requirements in times of water shortage, illumination devices (eg, flashlights with batteries, glow sticks, lanterns) to clinical staff in cases of power outages, and reversion to paper records when IT systems are out of commission. The key to a well-thought-out continuity plan is multiple levels of contingencies.

5. Communicate the urgency of these systems to service providers and verify priority restoration during response and recovery. Without formal agreements in place from telecommunications service and utility providers, priority restorations will be challenging. Hospital leaders should not make the assumption that hospitals receive the highest priority restoration without such an agreement in place.

6. Educate each department head, clinical leader, and key staff member on continuity plan roles. Providing training on, and reinforcing aspects of, the emergency management plan to key staff is critical to the success of the plan's implementation. Staff must understand their roles and responsibilities in regards to the plan, and to whom they report issues. This should be a key offering in the facility's emergency management-related training arsenal.

7. Conduct regular exercises involving mission critical systems. Facility leaders should work with their engineering and clinical staff to determine the best format and timing for exercises regarding mission critical systems. Tests of these systems can occur as stand-alone focused exercises or can be incorporated into a larger facility-wide exercise. The biggest focus should be on staff roles and responsibilities in times of critical outages or disruptions in service.

8. Use exercises and real experiences to learn about all mission critical systems' continuity plans. Given that evaluation is a critical aspect of exercises, hospital leaders and engineering staff should carefully consider all of the lessons learned from the exercises. Leaders should publicly note areas in which the facility or staff performed exceptionally well. Conversely, evaluators should carefully note opportunities for improvement and performance enhancement.

9. Review the continuity plan annually to ensure that it is up to date. The facility must closely examine its continuity planning efforts and documents. There are 2 main focus points for this review: First, as lessons learned from exercises or real events are discovered, the facility planning leadership should ensure that those opportunities for improvements are carefully addressed in the continuity plan. Second, because the facility's hazard vulnerability analysis must be updated every year to remain compliant with TJC requirements, the facility should also closely examine its continuity planning efforts and documents. As threats change, the plans must consistently evolve to address new and emerging threats.

Facility leaders often ask how they can assess the continuity plan's effectiveness. There are a number of process and analytical measures that could apply. To determine overall program effectiveness, leaders could monitor adherence to the above 9 steps. Most of the performance measures of mission critical systems are defined primarily by the TJC and $\mathrm{Na}$ tional Fire Protection Association. These standards provide an excellent framework for assessing their effectiveness. Other potential measures could include return on investment, levels of contingency plans, and evaluations of exercises or real events.

\section{About the Authors}

All of the authors are with Booz Allen Hamilton.

Address correspondence and reprint requests to Arnold B. Bierenbaum, Booz Allen Hamilton, One Preserve Pkwy, Rockville, MD 20852 (e-mail: bierenbaum_arnie@bah.com).

\section{Authors' Disclosures}

The authors report no conflicts of interest.

ISSN: 1935-7893 (C) 2009 by the American Medical Association and Lippincott Williams \& Wilkins.

DOI: 10.1097/DMP.0b013e31819e6d8f

\section{REFERENCES}

1. Florida Hospital Association. Eye of the Storm: Impact of the 2004 Hurricane Season on Florida Hospitals. Lessons Learned Information Sharing Web site. May 1, 2005. https://www.llis.dhs.gov/docdetails/details. do? content $I D=20161$. Accessed December 4, 2008.

2. International Strategy for Disaster Reduction and World Health Organization. Hospitals Safe From Disasters: Reduce Risk, Protect Health Facilities, Save Lives. 2008-2009 World Disaster Reduction Campaign brochure. Lessons Learned Information Sharing Web site. February 13, 2008. https://www. llis.dhs. gov/docdetails/details.do?content ID =30499. Accessed December 11, 2008.

3. Arendt LA, Hess DB. Hurricane Katrina, Volume I Emergency Response. Hospital Decision Making in the Wake of Katrina: The Case of New Orleans. Lessons Learned Information Sharing Web site. January 1, 2006. https://www.llis.dhs.gov/docdetails/details.do?contentID=17737. Accessed December 4, 2008.

4. Gray BH, Hebert K. Hospitals in Hurricane Katrina: Challenges Facing Custodial Institutions in a Disaster. Lessons Learned Information Sharing Web site. July 1, 2006. https://www.llis.dhs.gov/docdetails/details.do? contentID $=18379$. Accessed December 11, 2008. 